\title{
VOMES: a virtual organisation membership evaluation system
}

\author{
Junwei Cao* and Zhen Wang \\ Research Institute of Information Technology, \\ Tsinghua National Laboratory for Information Science and \\ Technology, Tsinghua University, \\ Beijing 100084, China \\ E-mail: jcao@tsinghua.edu.cn \\ E-mail: zhen-wang04@mails.tsinghua.edu.cn \\ *Corresponding author
}

\begin{abstract}
A grid supports geographically distributed resource sharing among multiple organisations, which can be considered as a special type of virtual organisations (VO). A cyberinfrastructure (CI) environment supports formation and management of multiple such VOs to meet various computing demands for researchers and scientists in different areas. How to found a trustable VO and aggregate reliable resources and users for the cross-domain collaboration in a $\mathrm{CI}$ becomes an important issue. In this work, a VO membership evaluation system (VOMES) is proposed to address this new challenge brought by the CI environment. VOMES includes a layered reputation system and a committee-based decision-making method to verify a new applicant to a VO and maintain corresponding VO trust levels. Simulation results show that our reputation system is accurate, robust and stable. VOMES can automatically help members in a CI environment establish and maintain VOs with high trust levels.
\end{abstract}

Keywords: cyberinfrastructure; CI; virtual organisations; VO; grid computing; trust management; reputation calculation.

Reference to this paper should be made as follows: Cao, J. and Wang, Z. (2012) 'VOMES: a virtual organisation membership evaluation system', Int. J. Networking and Virtual Organisations, Vol. 10, No. 1, pp.88-108.

Biographical notes: Junwei Cao is currently a Professor in Research Institute of Information Technology, Tsinghua University, China. He was a Research Scientist at the MIT and NEC Laboratories. He received his PhD in Computer Science from University of Warwick, UK, in 2001. His research interests are focused on distributed computing infrastructures. He has been working on cyberinfrastructure, grid and cloud computing, high performance computing applications for many years. He is a Senior Member of the IEEE Computer Society and a member of the ACM and CCF.

Zhen Wang is a graduate student of Research Institute of Information Technology, Tsinghua University, China. His research interests include virtual organisations for computing infrastructures, trust and reputation management for distributed computing. 


\section{Introduction}

Modern scientific research has great and various requirements for experimental instruments, computational ability and collaboration across organisations and subjects (Atkins et al., 2003). In order to support larger scale resource sharing, some grids no longer just focus on specific scientific applications but are expanded to handle multiple scientific research projects and meet various computing demands from researchers, such as Open Science Grid (OSG, http://www.opensciencegrid.org), Enabling Grids for E-science (EGEE, http://www.eu-egee.org/), TeraGrid (http://www.teragrid.org/) and so on. As these multifunctional grids are general platforms to provide computing support for various scientific applications, they are termed as cyberinfrastructure (CI) (Atkins et al., 2003; NSF Cyberinfrastructure Council, 2006; Fox, 2002; Znati, 2008). For one scientific project, a specific virtual organisation (VO) Holland (1998) and Pu et al. (2000) is proposed, established and managed in the CI. A VO is a set of users and resource providers (RPs) with agreements on usage purposes and sharing policies. As one of the most widely used grids, OSG supports more than 20 VOs for different scientific projects, ranging from biology to astrophysics, which are managed by different organisations, institutes or laboratories (OSG, http://www.opensciencegrid.org).

In a CI environment, one of the most important issues is how to establish a VO with appropriate users and RPs. In traditional grids, all users and RPs have been verified with each other in real world and enable their agreement on how to share resources on the network through certificates assigned by the same certificate authority (CA), which means they all belong to a same trust domain (Foster and Kesselman, 1998; Buyya et al., 2000). But in order to meet various requirements from scientific projects on computing resources, in a CI environment members (users or RPs) usually belong to different trust domains and have complex memberships with each other. One VO is established for one trust domain and one scientific application. Since CI members come from various fields and its number much more than that of grids, it is impossible for CI members to verify each other and establish trust relationship in real world.

Existing VO management tools, such as VOMS Monitoring Documentation (http://www.voms-monitor.grid.iu.edu/cgi-bin/index.cgi) and GUMS (https://www.racf.bnl.gov/Facility/GUMS/1.2/index.html), all focus on membership information services, without any automatic trust evaluation mechanism. To verify a new applicant, VOMS sends this application and self-description to a certain representative. The representative verifies the new applicant personally from several aspects, including past membership records and resource usage plans with following limitations (OSG, 2008).

- The procedure of verifying an applicant deeply relies on representative's individual experience and knowledge. As the member number in a CI environment (e.g., OSG) is always very large, it is impossible for representatives to get familiar with all the applicants.

- Since the judgment is given by an individual, the result could involve subjective factors. In this case, a tool is required to provide objective evaluation information to minimise subjective influence. 
- More representatives always mean more dissension but leads to a more accurate decision. Representatives with current tools can only consult manually with each other by e-mails, which is not efficient to meet the CI requirement.

- The registration based on e-mails and manual operations takes at least several days to complete one registering procedure and requires that representatives and $\mathrm{VO}$ administrators keep on line.

To address issue mentioned above, we propose VOMES based on a layered reputation system and committee-based fuzzy decision-making method. A reputation system is provided to track members' historical activities and use a reputation value (RV) to characterise their behaviours. The reputation system is in charge of recording all interactions in a CI environment. It calculates two kinds of reputation for a member, global reputation (GR) and local reputation (LR). GR calculation is based on the records of all interactions the given member involves, presenting general judgment to it. LR is calculated using interactions only between two specific members, which represent the judgment of one member on another. These two RVs can describe a member behaviour pattern (MBP) comprehensively and accurately in different aspects. A VO administrator can designate a committee of several representatives to verify the new applicant to the VO according to the reputation system and representatives' individual experiences. Every representative hands up their own judgment about the applicant according to the global and local RVs to the committee. The committee makes a decision after comprehensive consideration of opinions from every representative using a fuzzy-logic theory (Klir et al., 1997; Song et al., 2005; Zadeh 1994; Yano, 2001).

The rest of the paper is organised in the following way. In Section 2, reputation and trust managements in different virtual environments, e.g., e-commerce, peer-to-peer (P2P) and grids, are summarised. Section 3, provides detailed information on VOMES, including a reputation calculation system and a committee-based decision-making using fuzzy method. Simulation results to evaluate VOMES performance from various aspects are demonstrated in Section 4. We conclude our work and give a brief discussion on further work in Section 5.

\section{Related works}

There are three typical virtual communities on the internet, including e-comment, P2P and grids. How to characterise MBPs and help members establish appropriate trust relationships is a significant challenge for all virtual communities. Reputation system is wildly used to represent MBP and help members in virtual communities make decisions on interactions. Different communities have different policies and topologies, leading to different trust models and distinct ways of establishing trust relationships among members.

\subsection{Trust models for e-commerce}

E-commerce is a centralised, open and huge scale virtual community which requires high trust insurance and accurate and stable reputation system. As a trade platform for both consumers and good providers, e-commerce emphasises on providing accurate and simplified reputation schemes for consumers to conduct successful trades and inhibit 
malicious and cheating behaviours (Zacharia et al., 1999). Consumers can evaluate good providers according to their RVs (Yan and Kwei-Jay, 2008) and make a trade decision personally. How to provide accurate and stable reputation values (SRV) to characterise MBPs is a significant challenge for e-commerce administrators.

Generally speaking, trust models for e-commerce can be classified into two categories: accumulated models and average models. In an accumulated model, feedbacks from consumers on evaluation of providers are accumulated as the current RV. Positive feedbacks due to successful trades increase a provider's RV and negative ones decrease the value. This type of trust models cost little computational resources and storage; on the other hand, it ignores many other factors, for example successful trade rate and is not accurate enough. Some existing online markets, e.g., eBay (Dellarocas, 2001; Resnick and Zeckhauser, 2002), Yahoo! and TaoBao (Li and Liu, 2007), all adopt the accumulated trust model. The average trust model also accumulates all feedbacks of consumers on a provider, though divided by the number of feedbacks. The divided value is the average RV of the corresponding provider. The average model costs more and cannot separate different reputation levels of providers distinctly. Both Amazon and AuctionSoup adopt the average trust model.

\subsection{Trust models in P2P environment}

A P2P environment which usually focuses on resource discovering and data transferring applications is an open, self-organised and decentralised system. The trust relationship is also established based on member RVs and individual judgments. There is no central member to maintain member RVs due to the P2P topology. However, a GR evaluation is still required for judgment and inhibition of selfish and malicious behaviours. When a data transfer occurs between two members, a feedback to the file provider is also produced but maintained by the user. Since there is no central member with global information, P2P system usually adopts a mechanism to aggregate distributed feedbacks over the system to calculate GR of a certain member (Aberer and Despotovic 20001).

For a small scale P2P system, it is possible to collect feedbacks from all other members. But in most cases, a P2P system may include tens of thousands of members. How to choose members who can contribute their feedbacks about a given member and calculate all these feedbacks as the GR of that member becomes a key challenge. There are many mechanisms helping users to obtain feedbacks of most trustable members, such as powertrust (Zhou and Hwang, 2007), gossip trust [Zhoe et al., 2008; Zhou and Hwang, 2007] and eigentrust (Kamvar et al., 2003). In a P2P environment, trust models mainly are proposed to ensure the efficiency of collecting trustable members for feedbacks, for the collecting procedure would consume more time and storage as scales of P2P systems increase.

\subsection{Trust models in grid}

Once established, a grid environment is already a trustable and centralised virtual community (Grid Toolkits Project, http://www.globus.org; Cody et al., 2008). All members in a grid, users or RPs, already have achieved agreements on resource sharing before the grid is established. They maybe know each other very well in real world 
projects or collaborations. The grid is just an enabling technology for implementation of this achieved agreement and resource sharing policies on the internet. If a new member wants to join in a grid, he must be verified by grid representatives personally and manually. Since a grid is already a virtual community with high trustable and secure level and a new member is usually verified in real world, generally speaking, grid does not need a reputation system to evaluate its members.

\subsection{Trust models for $C I$}

$\mathrm{CI}$ is an open and partly-centralised virtual community that provides a public platform for establishing grid-like VOs and sharing resources among members dynamically. Members establish trust relationships through VOs and CI provides tools and services for $\mathrm{VO}$ management and maintenance. One VO forms one trust domain. Members can share resources with each other only if they belong to the same trust domain, i.e., in the same VO. One member can belong to different VOs for resource sharing, with different trust levels applied. Different VOs or trust domains can be established and maintained using VOMES, making it possible to satisfy various scientific demands while other virtual communities all have only one trust domain. Table 1 shows the difference from other virtual communities.

Table 1 Comparison of CI with other virtual communities

\begin{tabular}{lll}
\hline Virtual communities & \multicolumn{1}{c}{ Trust domains } & \multicolumn{1}{c}{ Trust relationships } \\
\hline E-commerce & $\begin{array}{l}\text { Members belong to the same } \\
\text { domain. }\end{array}$ & $\begin{array}{l}\text { Establish trust relationship only } \\
\text { between two members. }\end{array}$ \\
P2P & $\begin{array}{l}\text { Members belong to the same } \\
\text { domain. }\end{array}$ & $\begin{array}{l}\text { Establish trust relationship only } \\
\text { between two members. }\end{array}$ \\
Grid & $\begin{array}{l}\text { Members belong to the same } \\
\text { trust domain. }\end{array}$ & $\begin{array}{l}\text { All the members have trust relationships } \\
\text { among each other. }\end{array}$ \\
CI & $\begin{array}{l}\text { Members belong to different } \\
\text { trust domains. }\end{array}$ & $\begin{array}{l}\text { Members in the same VO have trust } \\
\text { relationships. }\end{array}$ \\
\hline
\end{tabular}

VOMES focuses on establish and maintain a specific trust domain for a given scientific project dynamically and is in charge of providing membership management services. VOMES provides a whole scheme including a reputation system and a committee-based fuzzy comprehensive decision making scheme to help CI members verify VO applicant to establish specific VOs for application specified requirements. Detailed information is given below.

\section{Virtual organisations membership evaluation system}

VOMES consists of two components: a layered reputation system and a committee-based fuzzy decision-making method. The reputation system associates RVs with MBPs and are also input values for the committee to make a decision. A committee is proposed to make a comprehensive decision on a given member according to RVs and VO configurations. 


\subsection{Key concepts}

\subsubsection{Task request time (TRT)}

Expected time a task should be completed on a given resource, denoted as $T r$. The user evaluates the complexity of the programme and resources assigned to him in order to estimate an expected execution time for his programme.

\subsubsection{Task completing time}

Task completing time (TCT) is the actual time from submitting the task to receiving the corresponding report. The report would be task outputs if the task is successfully completed or an error record if the task is failed. TCT value is denoted as $T c$.

\subsubsection{Interaction quality}

Denoted as $Q$ to represent three different situations: task is finished in time (i.e., $T c \leq T r$ ), beyond the request time (i.e., $T c>T r$ ) or task is failed, with different follow-up impacts on members. We assign different values to these three interaction quality (IQ) levels to encourage successful interactions and punish failures.

\subsubsection{Reference member}

This is a perfect member only exists in theory. Since the IQ is influenced by both participants, it is hard to ascribe a failure to either participant. The reference member $(\mathrm{RM})$ is an ideal member that provides perfect service to others. So IQ between a RM and any given member is only up to the real member, which can reflect its behaviour pattern independently.

\subsubsection{Member behaviour pattern}

We describe a MBP with three possibilities $P=\left\{p_{1}, p_{2}, p_{3}\right\}$, representing possibilities of three IQ levels, respectively.

\subsubsection{Reputation value}

After an interaction is finished, a score is calculated, according to the TRT and IQ of this interaction to present its impact to the reputation. The TRT $\times$ IQ value can be accumulated and divided by the sum of corresponding TRT, which is taken as the RV.

\subsubsection{Theoretical reputation value}

If we already know the actual MBP of a member, we can calculate an overall expectation value of its reputation, $Q P$, as the theoretical reputation value (TRV) of a member. We use the TRV to represent the MBP for simplicity in this paper. 


\subsubsection{Global reputation}

This reputation, denoted as $G r$, is maintained by the CI management centre (CIMC), tracking historical behaviours of a member since he joins in the CI. This reputation represents member interaction behaviour patterns with all other members in CI.

\subsubsection{Local reputation}

This reputation, denoted as is $L r$, is maintained by members themselves, recording historical interactions between specific members. This reputation represents one member's individual judgment about the behaviour pattern of a given member.

\subsubsection{Independent malicious behaviour (IMB)}

Members may decrease the quality of interactions deliberately. If an independent malicious member is a user, he may submit unexecutable tasks or unreasonable expected time $\operatorname{Tr}$ and so all. If it is a RP, he may not allocate sufficient resources and privileges to run the programme. This behaviour may disarrange ordinary interactions and the reputation system, which should be prohibited.

\subsubsection{Collusive malicious behaviour (CMB)}

Members cheat for a very high global RV by bogus successful interactions with collusive members. The two members may interact using empty tasks and result reports in order to cheat for high GR.

\subsection{Reputation calculation}

\subsubsection{GR calculation algorithms}

Assuming a member $M_{j}(j=1,2, \ldots \ldots, m$ ) (a user or RP) has interacted with other members for $n$ times, $Q_{i}(i=1,2, \ldots \ldots, n)$ is IQ of the $i^{\text {th }}$ interaction, and $T r_{i}$ and $T c_{i}$ are $\mathrm{T} R T$ and TCT of the $i^{\text {th }}$ interaction, respectively.

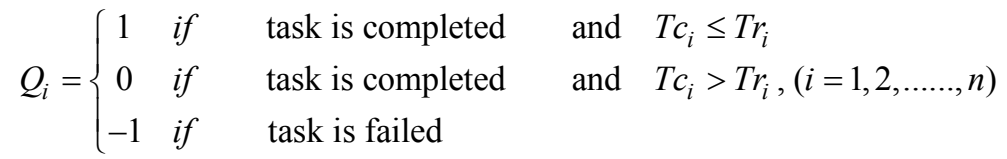

Given the definition of $Q$ above, we can calculate the TRV using the MBP of a member:

$$
T R V=Q P=[1,0,-1]\left[\begin{array}{l}
p_{1} \\
p_{2} \\
p_{3}
\end{array}\right]=p_{1}-p_{3}
$$

After $n$ interactions, the accumulated $Q$-weighted TRT for member $M_{j}$ is denoted as $V_{j}(n)$ while the accumulated TRT is denoted as $S_{j}(n)$. 


$$
\begin{aligned}
& V_{j}(n)=\sum_{i=1}^{n} \operatorname{Tr}_{i} * Q_{i},(j=1,2, \ldots \ldots, m) \\
& S_{j}(n)=\sum_{i=1}^{n} \operatorname{Tr}_{i},(j=1,2, \ldots \ldots, m)
\end{aligned}
$$

Because quality of interactions is determined by behaviour patterns of both participants, we use the switch calculator to approximate each of behaviour patterns, assuming they both have equal responsibility for the IQ. The validity of the algorithm is provided in Section 4 .

$$
G r_{j}(n)=\sqrt{\frac{V_{j}(n)}{S_{j}(n)}}=\sqrt{\frac{\sum_{i=1}^{n} T r_{i} Q_{i}}{\sum_{i=1}^{n} T r_{i}}},(j=1,2, \ldots \ldots, m)
$$

However, formula (5) does not consider the time factor: the latest interaction record can represent current behaviour pattern changes more accurately. We propose an attenuation index $\beta(0 \leq \beta \leq 1)$, to reflect the time influence on the record reference value. Considering time attenuation influence to the $\mathrm{GR}, \mathrm{Gr}_{j}(n)$ is calculated as follows:

$$
G r_{j}(n)=\sqrt{\frac{V_{j}(n)}{S_{j}(n)}}=\sqrt{\frac{\operatorname{Tr}_{n} Q_{n}+\beta V_{j}(n-1)}{T r_{n}+\beta S_{j}(n-1)}},(j=1,2, \ldots \ldots, m)
$$

Initial values of $V_{j}(0)$ and $S_{j}(0)$ are configured according to requirements when a member joins in CI.

\subsubsection{LR calculation algorithms}

LR is similarly defined as GR but only based on the past interactions between two specific members. $L r$ represents a member's individual judgment on a given member based on the interaction history between the two while $G r$ is the general judgment about a member of CI. We denote $\operatorname{Lr}_{j k}(n)$ as the LR the member $M_{j}$ assigns on $M_{k}$ at $n$ times interaction with $M_{k}$, which can be calculated similarly as follows:

$$
L r_{j k}(n)=\sqrt{\frac{T r_{n} Q_{n}+\beta V_{j k}(n-1)}{T r_{n}+\beta S_{j k}(n-1)}},(j, k=1,2, \ldots \ldots m)
$$

Compared with GR, LR is more flexible and personalisation. The local RV calculated by formula (7) is just for reference, since it is maintained locally by the member himself so can be changed personally. One member can assign a very low value to the LR of another member to prevent future interactions with that member. LR is designed to reflect individual judgment, compared with GR. 


\subsection{Membership evaluation}

In the last section, we propose a whole scheme to calculate global and LR to describe MBPs on different aspects. In general, an applicant should be approved by all existing members in the VO before he joins in. We cannot just simplify the issue by using uniform reputation thresholds to evaluate members, which is widely used in $\mathrm{P} 2 \mathrm{P}$ and e-commerce virtual communities, since different members may have different judgments for a given applicant. Another difficulty is that for both the applicant and VO members, it is not feasible to carry out the approval process between the applicant and every member because the member number of CI can be very large.

In this work, a committee-based fuzzy decision-making is proposed. The committee consists of part of members in a VO, called representatives, to present the various requests of all members and can reduce the approval process time. The fuzzy decision-making method can express fine-grained judgments of representatives compared with voting mechanisms (Venkaiah and Jalote, 1995; Yacoub et al., 2003) and make a comprehensive decision automatically, which dramatically simplifies the approval process.

The committee-based fuzzy decision-making method, including verification and reviews, are designed to insure the trust level of a VO and minimise malicious behaviours appearing aperiodically. New applicant has to be verified and members in the VO are reviewed periodically in order to prohibit possible malicious behaviours, for collusive malicious members may cheat in or the behaviour pattern of a member may change after joining. In this section, we introduce the committee-based fuzzy decision-making method in details and the performance of the two processes is evaluated in the next section.

\subsubsection{Fuzzy inference}

There are two reasons to adopt fuzzy inference. One reason is that RVs always have different meanings for different members, for example, a member with RV 0.7 means reliable enough for some users but may not for others. The membership vector can eliminate this difference and provide a uniform opinion description to the committee. Second reason is that fuzzy logic is robust to deal with uncertainty and inaccuracy of CI RVs. Fuzzy inference can map accurate and absolute RVs to membership degrees belonging to a fuzzy set using a function $\mu(x)(0 \leq \mu(x) \leq 1)$. The positive 1 presents fully belonging to the given set and 0 means nothing to do with the given fuzzy set.

Firstly we calculate a comprehensive RV, which is actually a weighted average of local and global RVs. Denote the comprehensive RV $M_{j}$ assigns on $M_{k}$ as $C r_{j k}$

$$
C r_{j k}=\alpha L r_{j k}+(1-\alpha) G r_{k}, 0 \leq \alpha \leq 1,
$$

where $\alpha$ is configured by $M_{j}$. The higher $\alpha$ is the more important $L r_{j k}$ is. In extreme cases that $\alpha$ equals to $1, M_{j}$ only trusts his own judgment. If $\alpha$ equals to $0, M_{j}$ totally trusts GR.

And then we use a membership function maps absolute comprehensive RVs to membership degrees to different sets. We divide members into three sets: reliable members (RLM), indeterminate members (IDM) and unreliable members (URM). The judgment $M_{j}$ makes on $M_{k}$ is described by the membership vector, denoted as $V_{j k}=\left(v_{1}, v_{2}, v_{3}\right)$, respectively representing membership degree $M_{j}$ regard $M_{k}$ as RLM, IDM and URM. 
A generally used membership function is provided to calculate the membership degree. Assuming membership function for $v_{1}, v_{2}$ and $v_{3}$ are $\mu_{1}\left(C r_{j k}\right), \mu_{2}\left(C r_{j k}\right)$ and $\mu_{3}\left(C r_{j k}\right)$. Figure 1 shows the three membership functions over $C r_{j k}$ and corresponding formulations are also included below.

Figure 1 Membership functions for fuzzy inference (see online version for colours)

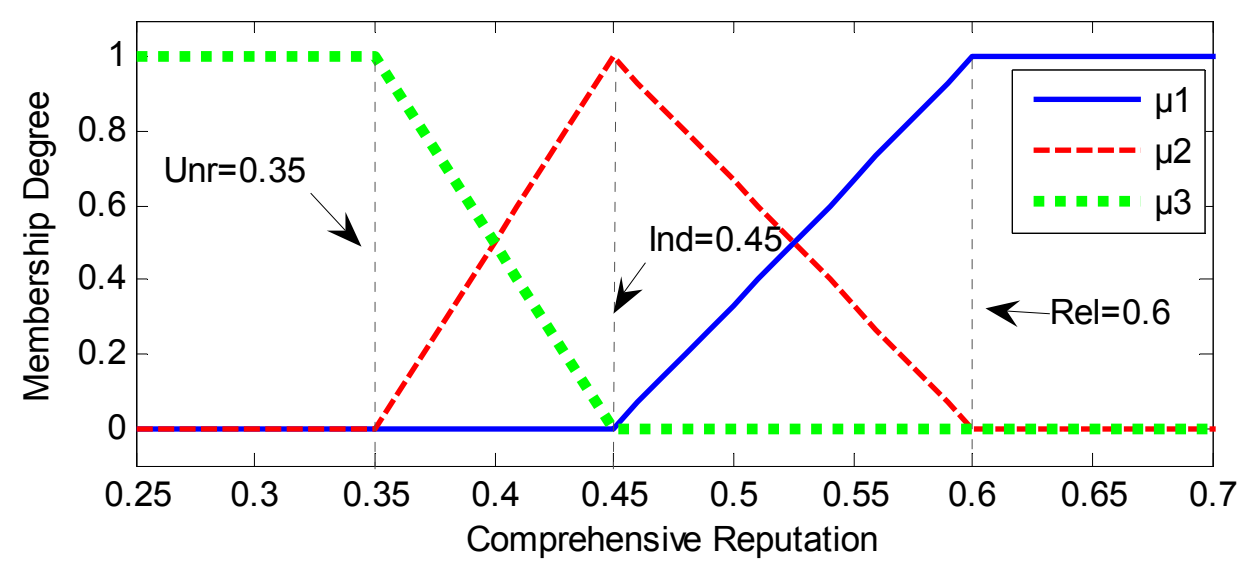

$$
\begin{aligned}
& \mu_{1}\left(C r_{j k}\right)=\left\{\begin{array}{cl}
0, & C r_{j k} \leq \text { Ind } \\
\frac{C r_{j k}-\text { Ind }}{R e l-\text { Ind }}, & \text { Ind }<C r_{j k}<\operatorname{Rel} \\
1, & C r_{j k} \geq \operatorname{Rel}
\end{array}\right. \\
& \mu_{2}\left(C r_{j k}\right)=\left\{\begin{array}{l}
0, \quad C r_{j k} \leq U n r \quad \text { or } \quad C r_{j k} \geq \text { Rel } \\
\frac{C r_{j k}-U n r}{I n d-U n r}, \quad U n r \leq C r_{j k} \leq \text { Ind } \\
\frac{R e l-C r_{j k}}{R e l-I n d}, \quad \text { Ind } \leq C r_{j k} \leq \text { Rel }
\end{array}\right. \\
& \mu_{3}\left(C r_{j k}\right)=\left\{\begin{array}{cl}
1, & C r_{j k} \leq U n r \\
\frac{I n d-C r_{j k}}{I n d-U n r}, & U n r<C r_{j k}<\text { Ind } \\
0, & \text { Rel } \leq C r_{j k}
\end{array}\right.
\end{aligned}
$$

In formula (9), Rel is the threshold for RLM while $U n r$ is the threshold for URM. Ind represents typical IDM. The membership degree vector $V_{j k}=\left\{\mu_{1}\left(C r_{j k}\right), \mu_{2}\left(C r_{j k}\right), \mu_{3}\left(C r_{j k}\right)\right\}$ can totally represent the opinion of the representative $M_{j}$ on the applicant $M_{k}$. A VO administrator receives all these judgments from representatives of the committee and integrates them to a final decision using the fuzzy comprehensive decision-making model described below. 


\subsubsection{Fuzzy comprehensive decision-making method}

There are two reasons for choosing the fuzzy comprehensive decision-making model: capability to handle uncertainty, fuzziness, and incomplete information adaptively and capability to make comprehensive decisions by integrating multiple factors (Shakouri and Menhaj, 2008; Chen and ye, 2008; Baldo and Zorz, 2007).

Assuming the number of representatives in the committee is $p$, the complete fuzzy comprehensive decision-making model consists of four elements: the factor set

$$
U=\left\{u_{l} \mid l=1,2 \ldots \ldots, p\right\}
$$

is a list of factors concerning with decision-making. In our case, every representative has influence on decision-making, so they are all regarded as factors. Corresponding weight vector

$$
A=\left\{a_{l} \mid l=1,2 \ldots \ldots, p\right\},
$$

can be used to describe different importance and influence of representatives in the committee: $a_{l}$ represents the weight of representative $u_{l}$ in decision-making. The evaluation set

$$
V=\left\{v_{k} \mid h=1,2,3\right\}
$$

represents three different sets members are divided into. The factor judgment vector

$$
f\left(u_{l}\right)=\left\{r_{l h} \mid h=1,2,3\right\}, \sum_{h=1}^{3} r_{l h}=1, l=1,2 \ldots \ldots, p,
$$

is membership degrees a member belongs to $v_{h}$, which is given by $M_{l}$.

We adopt the $M(\wedge \vee)$ model, namely the max-min model, to calculate the comprehensive decision. Let $\mathrm{B}$ be the judgment matrix:

$$
R=\left[\begin{array}{c}
f\left(u_{1}\right) \\
f\left(u_{2}\right) \\
\vdots \\
f\left(u_{p}\right)
\end{array}\right]=\left[\begin{array}{ccc}
r_{11} & r_{12} & r_{13} \\
r_{21} & r_{22} & r_{23} \\
\vdots & \vdots & \vdots \\
r_{p 1} & r_{p 2} & r_{p 3}
\end{array}\right]
$$

Use $M(\wedge \vee)$ model to calculate judgment vector $B$ and its normalisation $B^{\prime}$.

$$
\begin{aligned}
& B=A \circ R=\left\{b_{h} \mid h=1,2,3\right\} \\
& b_{l}=\underset{l}{\max \left(\min _{l}\left(a_{l}, r_{l h}\right)\right) \quad l=1,2, \ldots \ldots, p, h=1,2,3} \\
& b_{h}^{\prime}=\frac{b_{h}}{\sum_{h=1}^{3} b_{h}}, h=1,2,3
\end{aligned}
$$


$b_{h}^{\prime}$ represents the percentage degree the committee prefer the judgment of $v_{h}$. The VO administrator can configure a threshold for $b_{h}^{\prime}$ : if there is a $b_{h}^{\prime}$ higher than the threshold, the VO administrator will adopt the decision $v_{h}$, since he believes this decision can represent the majority view of the committee or VO members. If there is no $b_{h}^{\prime}$ higher than the threshold, the VO administrator will check the applicant personally.

\section{Simulations and evaluations}

In this section, we evaluate the accuracy and robustness of reputation calculation and the performance of verification procedure and review procedure.

\subsection{Reputation analysis}

\subsubsection{Performance metrics}

A good and robust reputation system should rapidly and exactly reflect MBPs, has distinct degrees to differentiate various behaviour patterns and can eliminate malicious behaviours. We define three performance metrics, including reputation standard variance (RSV), SRV and rising time (RT) over different behaviour patterns and different percentages of malicious members (PMM), consisting of percentage of independent malicious members (PIMM) and percentage of collusive malicious members (PCMM).

Figure 2 Dynamic processes of reputation calculation - performance metrics (see online version for colours)

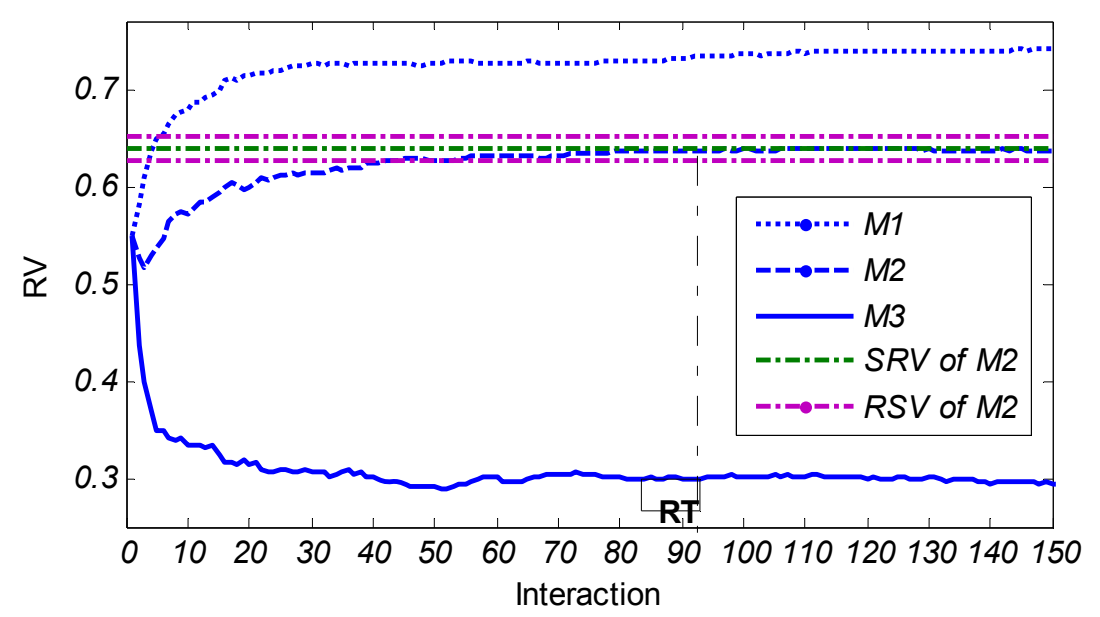

Figure 2 shows how reputation changes over interactions in a VO with 100 members and $5 \%$ malicious members. Three curves respectively represent members with three different behaviour patterns: reliable, unreliable and an ordinary member between the two extreme cases. The TRV of three members are $0.9,0.7$, and 0.3 , respectively. Since interactions in a simulated VO environment are all based on probability, all the data is an average value of 100 simulation results in this section. 
As shown in Figure 2, the $R T$ is defined as the first time the reputation reaches the $S R V$. $R T$ reflects the rate RV reflect the MBP. The faster the reputation reaches the stable value, the more sensitive the reputation system is. However, higher sensitivity of the reputation system always leads to unstable RVs. The RV may oscillate dramatically near the $S R V$ though the MBP is fixed. $R S V$ is used to evaluate the stableness of the reputation system. Three curves final reach stable values, which we hope can reflect actual TRV. $S R V$ is used to analyse the reputation accuracy.

From the simulation, the rise time $R T$ varies little over different behaviour patterns but is greatly influenced by initial values of $V_{j}(0)$ and $S_{j}(0)$. Because in most cases, statistic initial values for all the members are fixed in CI, so the $R T$ changes a little and we just focus on analysing $R S V$ and $S R V$ changes over different situations below.

\subsubsection{Simulation environment}

Our simulation environment is a VO with 100 members with TRV from 0.6 to 0.75 . The malicious member is defined as the member that has distinct low TRV from 0.2 to 0.3 . We put a member with assigned behaviour pattern in the VO and track its GR after every interaction. In the experiment of analysing the performance over different behaviour patterns, the percentage of malicious members is fixed at 5\% and the TRV of tracked member changes from 0.2 to 1 . In the experiment of analysing the performance over different PMM, TRV of tracked member is fixed at 0.7 and the percentage of malicious members changed from 0 to $50 \%$.

Because both independent and collusive malicious members have low TRV and the only difference is that collusive malicious members cheat for high GR through bogus interactions with accomplice, we just consider how the low TRV affects the reputation system and the issue of bogus high GR is addressed in Section 4.2. As the LR involves too much subjective factors and has similar calculation formula as the GR, we just analyse performance metrics for GR in the following sections.

\subsubsection{Performance over different behaviour patterns}

As shown in Figure 3, it is obvious that $R S V$ is limited to sufficient low values, no matter TRV is. RVs of malicious members (with low TRV) have higher $R S V$ which indicates that their RV change more easily. This is because malicious members always have low RVs and are easily influenced by randomicity of IQ.

Figure 3 Performance evaluation - $R S V$ over $T R V$ (see online version for colours)

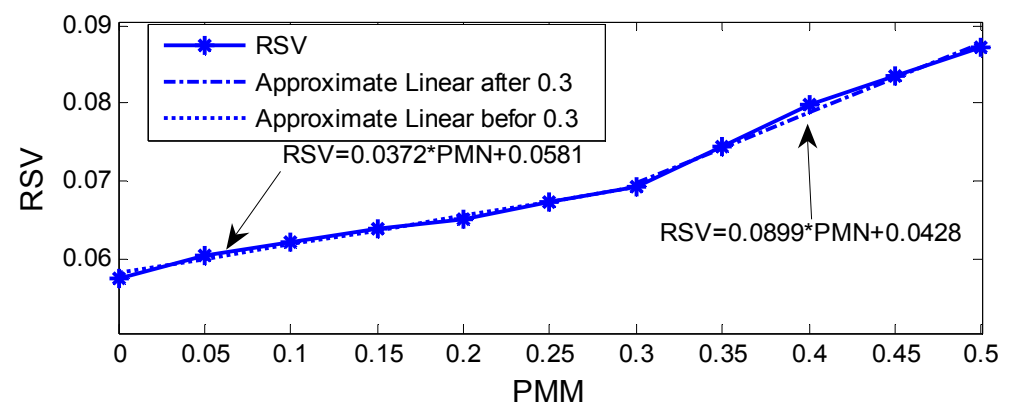


RVs should also reflect actual behaviour patterns of members, which can be evaluated using $S R V$. Figure 4 shows three curves, the global $S R V$ calculated by the reputation system, linear approximation for the calculated $S R V$ and the actual TRV.

Figure 4 Performance evaluation - SRV over $T R V$ (see online version for colours)

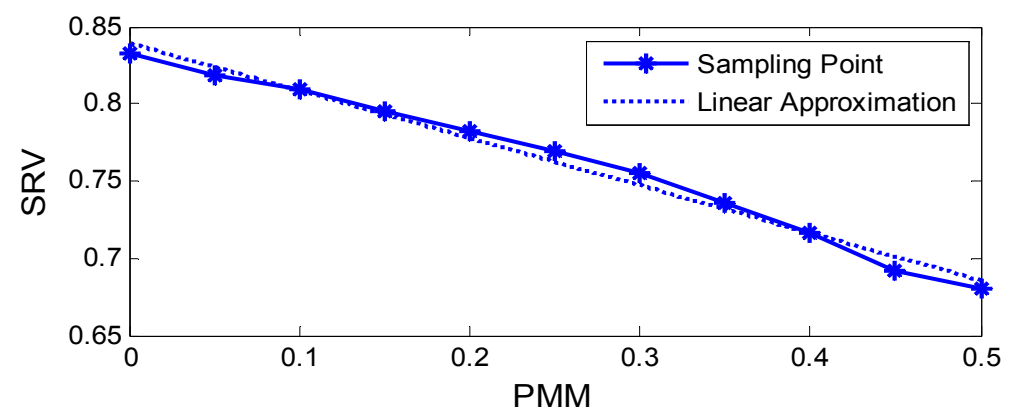

It is obvious that the calculated $S R V$ is an approximate linear line. Linearly mapping the actual TRV to $S R V$ can insure the RV having sufficient differential degree to classify members with different behaviour patterns. The linearity relationship between $S R V$ and TRV can ensures the $S R V$ accuracy and differential degrees.

\subsubsection{Performance over different PMM}

Another performance aspect of reputation calculation is robustness to malicious behaviours. Malicious members lead to failed interactions which decrease and disorder RVs of actual RLM. A good reputation calculation scheme should minimise influence of malicious behaviours to a reasonable scope and ensure stableness and accuracy of RVs of other members.

Figure 5 Performance evaluation - RSV over $P M M$ (see online version for colours)

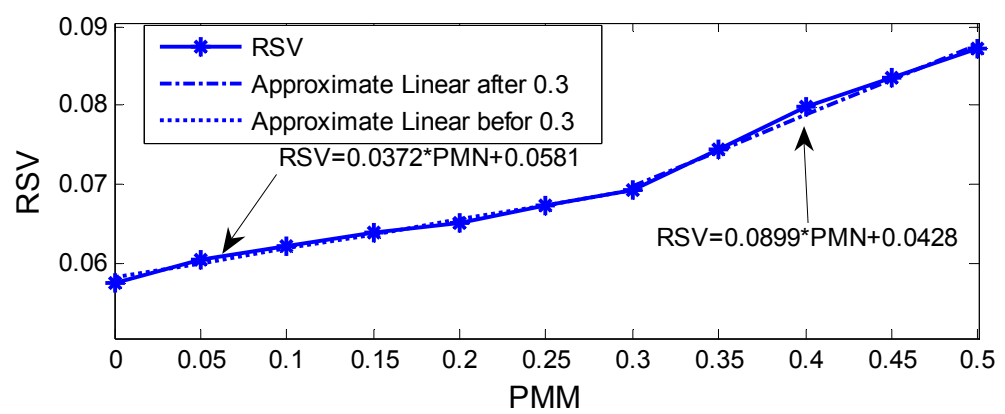

Figure 5 shows how the $R S V$ of a member with $T R V=0.7$ changes over different $P M M$ in the VO. It is shown in the figure that $R S V$ increases linearly as the $P M M$ increases. There is a turning point at $P M M=0.3: R S V$ increases faster if $P M M$ is bigger than $30 \%$. So it is better to keep $P M M$ in a VO below $30 \%$. When $P M M$ equals to $50 \%$, the $R S V$ reaches the maximal point. 
Figure 6 Performance evaluation - SRV over PMM (see online version for colours)

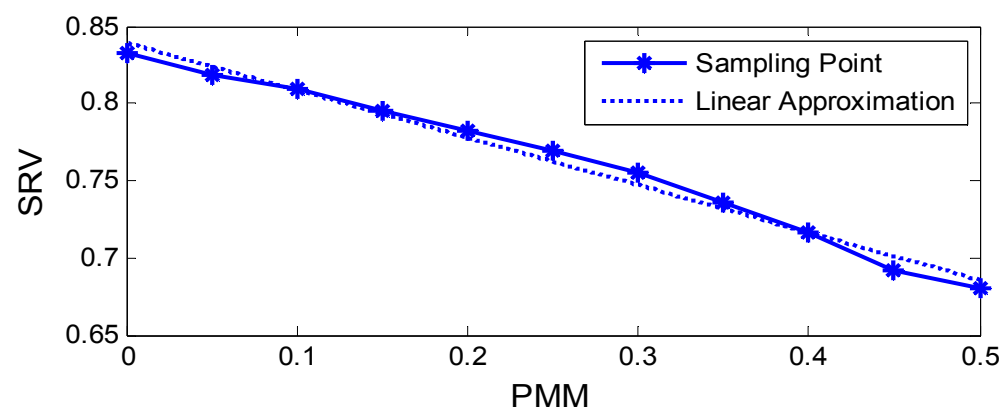

Figure 6 shows how malicious members affect the $S R V$. The $S R V$ decreases linearly when $P M M$ increases and the slope is -0.27 . $S R V$ keeps stable if there are only a small number of malicious members in the $\mathrm{VO}$ and the verification procedure can limit the percentage of malicious members in a VO, which is introduced in Section 4.2.

In this section, we evaluate the reputation system using three performance metrics, $R S V, S R V$ and $R T$. We examine how $R S V$ and $S R V$ changes over different TRV and PMM in CI. From the results, we can conclude that our reputation calculation system can linearly, rapid and stably reflect various MBPs and greatly defence malicious members in a VO with no more than $30 \%$ PMM.

\subsection{Committee performance}

Verification occurs when an applicant joins in the VO and reviews are carried out periodically to identify both independent and collusive malicious members and maintain the VO trust level in time. In this section, we analyse the performance of the two processes over different $P M M$. The committee has great influence on the verification performance. So we also test how the scale of the committee affects the verification performance in Section 4.2.5.

\subsubsection{Performance metrics}

Two performance metrics are used to evaluate performance of verification and reviews: errors of classifying malicious members as reliable ones and errors of classifying RLM as malicious ones. We define the two types of errors as positive errors $(P E)$ and negative errors $(N E)$ respectively.

$P E=($ Number of malicious members regarded as reliable $) /$

(Number of malicious members)

$N E=($ Number of RLM regarded as malicious) $/$ (Numberof RLM).

\subsubsection{Simulation environment}

The actual environment is always unstable, in which MBP changes aperiodically and RV always locates in the transition phase from the initial to SRV. A series of interactions among members are simulated in CI before the experiment starts to make all member 
RVs transient. Because we assume in the environment MBP do not change as the interaction happens, we keep all the members staying in the transition phase by controlling the number of interactions before the experiment.

In the environment, both independent and collusive malicious members have to be identified. Both of them have low TRV and the only difference is that collusive malicious members have high bogus global RVs but independent malicious member do not. Two types of reputation, GR and LR are calculated as input values of committee-based fuzzy decision-making method. Detailed experiment is shown in Figure 2.

Table 2 Simulation parameters

\begin{tabular}{lccc}
\hline$m$ & 200 & Ind & 0.6 \\
\hline$p$ & 10 & Number of members in CI & 1,000 \\
$\alpha$ & 0.4 & Reliable MBP & $(0.9: 0.067: 0.33)$ \\
$\beta$ & 0.99 & Malicious MBP & $(0.7: 0.2: 0.1)$ \\
Rel & 075 & Initial value $V_{j}(0): S_{j}(0)$ & $3: 10$ \\
$U n r$ & 0.67 & Collusive malicious member & $9: 20$ \\
& & Initial value $V_{j}(0): S_{j}(0)$ & \\
\hline
\end{tabular}

\subsubsection{PE and NE of verification}

The GR of independent malicious members decreases with interactions while collusive malicious members may cheat for high GR. But local RVs assigned to both types of malicious members by representatives decrease to reflect actual behaviour patterns if malicious members interact with representatives. Our fuzzy comprehensive decision-making can aggregate the two kinds of information from all representatives in a committee and make a decision according to membership vectors and weight vector automatically. In the experiment, the committee verifies all the members in CI and classifies them into three types: reliable, unreliable and indeterminate. We hope the committee can classify the member to the corresponding sets: actual malicious members into unreliable class, the members with high TRV into reliable class and the members between the two into indeterminate class which need to be further verified.

Figure 7 Performance evaluation $-N E / P E$ over $P M M$ (see online version for colours)

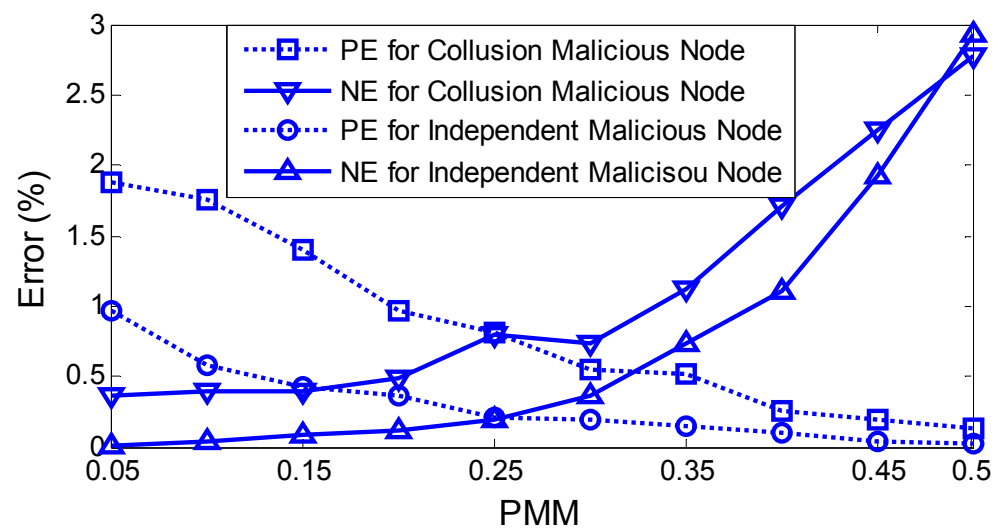


Figure 7 shows the $P E$ and $N E$ curves over different $P I M M$ and $P C M M$. From that figure, it is obvious that the committee can verify most of malicious members and RLM, for $P E$ is no more than 0.02 while $N E$ is lower than 0.03 . Collusive malicious members may have high GR by giving high feedbacks with each other. This high bogus GR may cheat the committee to approve actual malicious members. In this case, LR representatives assigned on the given member should be considered. The final decision is based on the weighted average of GR and LR. As illustrated in Figure 7, it is more difficult to identify collusive malicious members from RLM than independent malicious members, which makes higher $P E$ and $N E$ values. But $P E$ and $N E$ for collusive malicious behaviours (CMBs) are still low enough. No matter how strict the verification is, we can never ensure that all malicious members are identified and rejected when applicants reach the VO committee. So performance of periodical reviews is also of importance.

\subsubsection{PE and NE of review}

This experiment is carried out based on a VO environment consists of three types of members: RLM, independent and collusive malicious members. Every review selects some members that are regarded as malicious ones, which may contains both actual malicious members and RLM. $P E$ and $N E$ values after ten rounds of reviews are calculated respectively to evaluate its performance. Since most of independent malicious members are rejected during verification and are much easier to be identified than collusive malicious members, we only consider review performance of identifying collusive malicious members from reliable ones in the VO. In this experiment, reviews occur once every 50 interactions.

Figure 8 Performance evaluation $-N E / P E$ over $P C M M$ (see online version for colours)

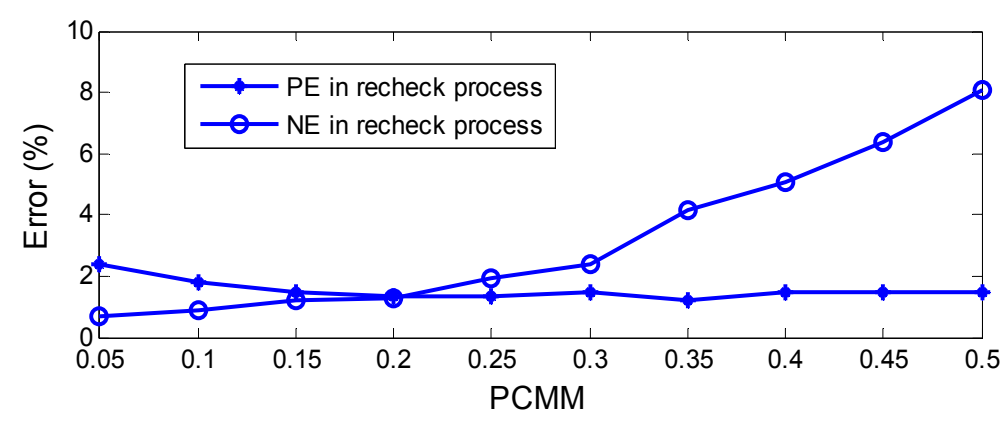

Figure 8 shows curves of $P E$ and $N E$ over different number of collusive malicious members. It is obvious that $P E$ is stably limited to a very low level, which means most of collusive malicious members can be identified from RLM, no matter how many collusive malicious members there are. $N E$ increases with the number of collusive malicious members. That is because the malicious may bring failed interactions with RLM, which decreases global RVs of both sides and disorders other VO regular interactions. However, the number of RLM regarded as malicious members is $1.93 \%$ at most if the number of malicious members is no more than 50 .

In this section, we evaluate performance of committee-based verification and reviews. Two performance metrics, $P E$ and $N E$, are proposed to evaluate accuracy and effectiveness of the two processes. From simulation and analysis results, it can be 
concluded that both processes can identify the two types of malicious members from RLM with very low $P E$ and $N E$ values. Collusive malicious members are more difficult to be identified compared with independent ones. Considering the behaviour pattern changes after a member joins in a VO, we need reviews to trace MBPs periodically and remove malicious ones in real time. Verification ensures only small number of malicious members have probability to cheat in the VO while reviews are essential to maintain the VO trust level. Simulation results show that the combination of verification and reviews ensure that only RLM can be the VO member at any time.

\subsubsection{PE and NE of different committees}

The number of representatives in a committee has significant impact on the classification performance. Intuitively, more representatives are in the committee, more accurate the verification of the committee is, since more knowledge about an application is collected by the committee from representatives. It is quite similar with that in practical world. In this section, we test the effectiveness of the representatives through a simulated experiment.

In the experiment, the $\mathrm{CI}$ and $\mathrm{VO}$ environment configurations are listed in Table 2, assuming $200 \mathrm{VO}$ candidates requiring to be verified. We assume there are 50 collusive malicious members in these 200 candidates. Verification performances of different scales of committees are shown in Figure 9.

Figure $9 P E$ and $N E$ of different number of representatives (see online version for colours)

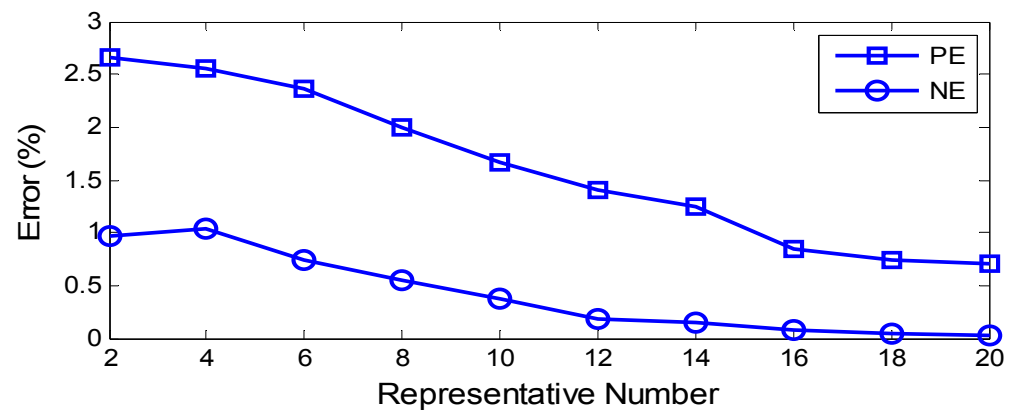

As show in Figure 9, verification errors, $P E$ and $N E$ decrease as the representative number increases. That is to say that expanding the scale of the committee in a VO can enhance the verification accuracy for a new applicant. And a new representative joining into the committee always has an obvious positive effectiveness for the CAVM performance. It is an expandable way to enhance the verification accuracy if the VO administrator or other members have high secure requirements.

But it does not mean that we can expand the committee without any limitation to continuously enhance the verification accuracy. Two reasons impede the extension. The first one is that it is infeasible to select large number of trustable and experienced nodes from CI as representatives, for the knowledge of the VO administrator is limited. Generally, only a few numbers of members are trusted by the VO administrator and empowered as representatives to verify a new applicant. Another reason is that expanding the scale of the committee will increase the complexity of the verification process. If $P E$ 
and $N E$ are lowered to an acceptable scope, it is unnecessary and inefficient to increase the number of representatives in the committee.

\section{Discussions}

VOs are considered as the major form to manage large-scale distributed systems in future. Trust and reputation management for virtual organisations is essential functionalities for membership management. For example, how to detect malicious members from good members with high reputation is a quite important issue. In this work, a committee-based approach is proposed for VO membership management. A simulation environment is implemented to enable quantitative analysis of committee performance.

Committee members are representatives of all members in a VO. A fuzzy decision making method is adapted in this work to aggregate various evaluation results so that reasonable performance of membership evaluation can be achieved. There are many factors that could have impact on performance of the committee in a VO. In this work, we evaluate the relationship between the number of committee members and classification performance and some preliminary results are achieved.

There are many similar requirements in grid, P2P, and e-commerce systems. Our approach is unique since members are evaluated using a committee. The results are not only useful for trust management and credit calculation. For example, in a grid computing environment, if better resources that can provide better services can be distinguished from others, better grid resource management and scheduling can be achieved.

\section{Conclusions}

In this paper, we propose a VOMES, consisting of a layered reputation system and a committee-based fuzzy comprehensive decision-making method, to help users and RPs establish a VO with insurance of trustiness and robustness. Simulation experiments are carried out to analyse and evaluate the proposed VOMES using different aspects of performance metrics. The contributions of VOMES are summarised as follows:

- A layered reputation system. There is no existing work addressing reputation and trust issues in CI currently. Our reputation system can rapidly, stably and accurately reflects actual behaviour patterns of VO members and provides significant differential degrees to classify different behaviour patterns.

- Committee-based fuzzy decision-making. A committee-based decision-making method is proposed in our work. Experienced representatives in the committee represent various requests of the whole VO to verify new applicants and review memberships periodically. This mechanism obviously reduces malicious behaviours and maintains the VO trust level automatically. Besides that, $\mathrm{VO}$ administrator can simply enhance the accuracy of the verification through extending the committee scale, namely empower new member as the representative to join in the decision-making process. 
- Membership reviews. Because the committee-based fuzzy decision-making method is carried out automatically, it is possible to review the memberships in a VO periodically. It can maintain the VO trust level in real time and obviously identify malicious behaviours.

The above components and method makes VOMES stably and accurately verify new applicant, defeat potential independent and CMB and expansive to enhance its performance. Currently, performance of VOMES is analysed and evaluated in a simulation environment. Future works include deployment of VOMES in real-world grid and CI environments so that performance of VOMES can be further improved.

\section{Acknowledgements}

This work is supported by National Science Foundation of China (grant No. 60803017) and Ministry of Science and Technology of China under National 973 Basic Research Program (grants No. 2011CB302505 and No. 2011CB302805).

\section{References}

Aberer, K. and Despotovic, Z. (2001) 'Managing trust in a peer-2-peer information system', Proceedings of 10th International Conference on Information and Knowledge Management, 2001.

Atkins, D.E. et al. (2003) 'Revolutionizing science and engineering through cyberinfrastructure, national science foundation blue - ribbon advisory panel on cyberinfrastructure', January 2003.

Baldo, N. and Zorz, M. (2007) 'Cognitive network access using fuzzy decision making', IEEE International Conference on Communications, pp.6504-6510.

Buyya, R., Abramson, D., Giddy, J. and Nimrod, G. (2000) 'An architecture for a resource management and scheduling system in a global computational grid', Proceedings of 4th International Conference/Exhibition on High Performance Computing, May 2000, pp.283-289.

Chen, H. and Ye, Z. (2008) 'Research of P2P trust based on fuzzy decision-making', Proceedings of 12th International Conference on Computer Supported Cooperative Work in Design, pp.793-796.

Cody, E. et al. (2008) 'Security in grid computing: a review and synthesis', Decision Support Systems, pp.749-764.

Dellarocas, C. (2001) 'Analyzing the economic efficiency of ebay-like online reputation reporting mechanisms', Proceedings of 3rd ACM Conference on E-Commerce, 2001.

Foster, I. and Kesselman, C. (1998) 'The grid: blueprint for a new computing infrastructure', Morgan Kaufmann, San Francisco.

Fox, G. (2002) 'E-science meets computational science and information technology', Computing in Science \& Engineering, July-August 2002.

Holland, C.P. (1998) 'Business trust and the formation of virtual organizations', Proceedings of 31st Hawaii International Conference on System Sciences, January 1998, pp.602-610.

Kamvar, S.D., Schlosser, M.T. and Garcia-Molina, H. (2003) 'The eigentrust algorithm for reputation management in $\mathrm{p} 2 \mathrm{p}$ networks', Proceedings of 12th International Conference on World Wide Web.

Klir, G.J., St. Clair, U. and Yuan, B. (1997) Fuzzy Set Theory: Foundations and Applications, Prentice Hall. 
Li, Q. and Liu, Z. (2007) 'Research on Chinese C2C e-business institutional trust mechanism: case study on Taobao and eBay, international conference on wireless communications', Networking and Mobile Computing, pp.3787-3790.

NSF Cyberinfrastructure Council (2006) NSF's Cyberinfrastructure Vision for 21st Century Discovery, Version 7.1., National Science Foundation, July 2006.

Open Science Grid (OSG) (2008) 'OSG VO registration policy and procedure', OSG DocDB document 737v2, available at http://www.opensciencegrid.org, 14 April.

Pu, S.Y., Lee, M.K.O., Yi, L.S. (2000) 'Virtual organizations: the key dimensions', Proceedings of Academia/Industry Working Conference on Research Challenges, April, pp.27-29.

Resnick, P. and Zeckhauser, R. (2002) 'Trust among strangers in internet transactions: empirical analysis of eBay's reputation system', The Economics of the Internet and E-Commerce.

Shakouri, H.G. and Menhaj, M.B. (2008) 'A systematic fuzzy decision-making process to choose the best model among a set of competing models', IEEE Transaction on Systems, Man and Cybernetics Part A, September 2008.pp.1118-1128.

Song, S., Hwang, K., Zhou, R. and Kwok, Y-K. (2005) 'Trusted P2P transactions with fuzzy reputation aggregation', IEEE Internet Computing, November December 2005.

Venkaiah, D. and Jalote, P. (1995) 'An integer programming approach for assigning votes in a distributed system', Proceedings of 14th Symposium on Reliable Distributed Systems, pp.128-134.

Yacoub, S., Lin, X., Simske, S. and Burns, J. (2003) 'Automating the analysis of voting systems', Proceedings of 14th International Symposium on Software Reliability Engineering, pp.203-214.

Yan, W. and Kwei-Jay, L. (2008) 'Reputation-oriented trustworthy computing in e-commerce environments', IEEE Internet Computing, pp.55-59.

Yano, H. (2001) 'An interactive fuzzy decision making method for decentralized multiobjective programming problems', IFSA World Congress and 20th NAFIPS International Conference, pp.98-103.

Zacharia, G., Moukas, A., Maes, P. and Media Lab. (1999) 'Collaborative reputation mechanisms in electronic marketplaces', Proceedings of 32nd Annual Hawaii International Conference on System Sciences, 1999, p.7.

Zadeh, L.A. (1994) 'Fuzzy logic, neural networks and soft computing', ACM Communications, Vol. 37, No. 3, pp.77-84.

Zhou, R. and Hwang, K. (2007) 'Gossip-based reputation aggregation for unstructured peer-to-peer networks', Proceedings IEEE International Symposium of Parallel and Distributed Processing, pp.1-10.

Zhou, R. and Hwang, K. (2007) 'Powertrust: a robust and scalable reputation system for trusted peer-to-peer computing', IEEE Transactions on Parallel and Distributed Systems.

Zhou, R., Hwang, K. and Cai, M. (2008) 'GossipTrust for fast reputation aggregation in peer-to-peer networks', IEEE Transactions on Knowledge and Data Engineering.

Znati, T. (2008) 'Challenges and future research directions in large-scale complex systems', Proceedings of 28th International Conference on Distributed Computing Systems, June 2008, pp.17-20. 